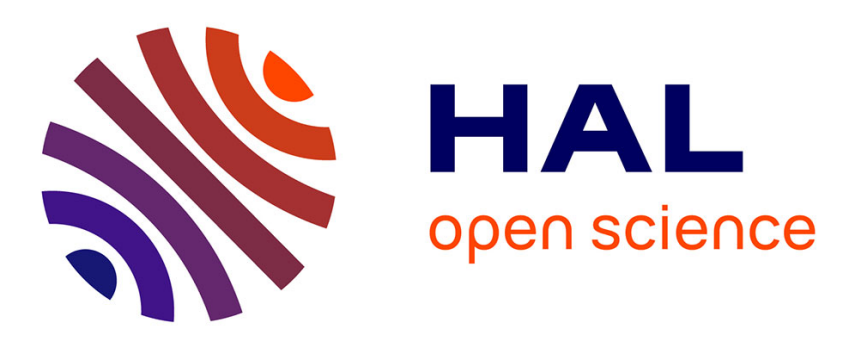

\title{
Mean-field limit of the fixed-reward incentive mechanism in Delay Tolerant Networks
}

\author{
Thi Thu Hang Nguyen, Olivier Brun, Balakrishna Prabhu
}

\section{To cite this version:}

Thi Thu Hang Nguyen, Olivier Brun, Balakrishna Prabhu. Mean-field limit of the fixed-reward incentive mechanism in Delay Tolerant Networks. 16th Symposium on Modeling and Optimization in Mobile, Ad Hoc and Wireless Networks (WiOpt 2018), May 2018, Shanghai, China. 10.23919/WIOPT.2018.8362810 . hal-02062180

\section{HAL Id: hal-02062180 \\ https://hal.laas.fr/hal-02062180}

Submitted on 8 Mar 2019

HAL is a multi-disciplinary open access archive for the deposit and dissemination of scientific research documents, whether they are published or not. The documents may come from teaching and research institutions in France or abroad, or from public or private research centers.
L'archive ouverte pluridisciplinaire HAL, est destinée au dépôt et à la diffusion de documents scientifiques de niveau recherche, publiés ou non, émanant des établissements d'enseignement et de recherche français ou étrangers, des laboratoires publics ou privés. 


\title{
Mean-field limit of the fixed-reward incentive mechanism in Delay Tolerant Networks
}

\author{
Thi Thu Hang Nguyen ${ }^{1,2}$, Olivier Brun ${ }^{2}$, and Balakrishna J. Prabhu ${ }^{2}$ \\ ${ }^{1}$ Université de Toulouse, INSA, Toulouse, France \\ ${ }^{2}$ LAAS-CNRS, Université de Toulouse, CNRS, Toulouse, France
}

\begin{abstract}
We investigate the asymptotic performance of a reward incentive Delay Tolerant Network based on mean field limit. We consider a two-hop network with one source and one destination and $N$ relays. The source is backlogged and sends messages to the destination by forwarding to the relays it meets. For each message, there is a promised reward for the first one who successfully transmits it to the destination. It was shown in a previous work, the optimal policy for the relays is of thresholds type (a relay will accept a message until certain time and drop it after a second threshold). When the second threshold in infinite, we give the mean-field ODE and show that all the messages have the same probability of success. When the second threshold is finite we only give an ODE approximation since the dynamics are not Markovian.
\end{abstract}

\section{INTRODUCTION}

Delay Tolerant Networks (DTNs) rely upon mobility of nodes to ensure connectivity of the network. In DTNs, nodes play the role of relays who can receive messages from sources, store and forward them to other nodes, until the message arrives to the destination. When a relay can forward a message to another relay, the DTN is a known as multi-hop network because a message can be relayed by multiple nodes before arriving at the destination [1], [2], [3], [4]. While this mechanism improves the probability of success and reduces the time to deliver a message, it also increases energy consumption of the relays. In order to reduce energy consumption, a two-hop mechanism in which a relay is not allowed to forward to others was considered in [5], [6], [7], [8], [9], [10].

Relaying of messages by nodes is essential in DTNs to maintain connectivity and to keep the network alive. On the other hand, nodes incur costs for transmitting, storing, and receiving messages when they participate in relaying them. It would be tempting for a node to avoid these costs by not participating while benefiting from the relaying services of other nodes. In order to discourage such selfish and harmful behaviour of nodes and to solve the free rider problem, several incentive mechanisms have been proposed in the literature. For most of these mechanisms, the analysis of the performance measures such as the mean delivery time or the probability of success is complex, and it is not easy to figure out how much reward should be proposed by the source to achieve a given performance.

An exception to these complex mechanisms is the one proposed in [10]. In there, the source proposes a fixed reward for the delivery of each message. The first relay to deliver this message gets this reward. In [11], [12], this mechanism was analysed for a backlogged source and strategic relays who were allowed to accept or not messages and allowed to drop them whenever they wanted. Using the framework of stochastic games, it was proven that the equilibrium strategy of a relay has two thresholds. That is, for each message, relays accept this message only until a first threshold. Once a relay has accepts a message, it keeps it until a second threshold unless it meets the destination earlier. Conditions for the existence of a strictly positive first threshold as well as for a finite second threshold were also given. The structure of the equilibrium strategy was then used to derive the relationship between the reward proposed and the performance measures. The advantage of this fixed-reward mechanism is that in some cases this relationship can be numerically computed based on a recursive formula thereby giving a simple way to compute the rewards that the source must propose to achieve a targeted performance.

\section{A. Contributions}

In this paper, we pursue the analysis of the fixed-reward mechanism further by investigating the mean-field limit of the system dynamics when the number of relays becomes large. The backlogged source proposes a message until no relay will accept it any more after which it proposes the next message. It will be shown that the time-scale for the duration of a message being proposed by the source, that is, the first threshold, is $O(1 / N)$ where $N$ is the number of relays. The second threshold will be shown to be $O(1)$. We shall focus the analysis on the case of the second threshold being infinite. This leads to a Markovian description of the system, and to the mean-field limit. We shall give the necessary and sufficient that the reward must satisfy in order for the second threshold to be infinite. We let the number of messages is proportion to the number of relays, say $k=t N$. We then let $N$ tend to infinity and study the limits. In order to do that, we consider a mean field interaction model with two states: state 1 for having a message and state 0 otherwise. We will show that:

- When the second threshold is infinite, we shall show the converge to an ODE and give its solution.

- In this case, the probability of success and the delay in limit do not depend on $t$. 
- When the second threshold is finite, since the dynamics are no longer Markovian, we give an approximation for the mean field limit.

\section{B. Related works}

In literature, there has been many researches about meanfield analysis for Delay Tolerant Networks. For instance, [13] considers a network with one source and one destination. In this network, the source has only one message to send during a time $T$ (the life time of a message). The author uses multi-hop setting where a node will consider to forward a message to other node based on the available energy. He does not consider any incentive scheme but work with the trade-off between the delay and the energy which gives us a complicated optimal problem. In contradiction, mean-field leads to a simpler result with a threshold which depends on the remain energy. [14] works in the mean-field scheme of a Delay Tolerant Network in which the source wants to send an information formed by $K$ packages. The destination sends feedback to the source (via relays) about the number of packages have not received yet, based on that, the source will send again some corresponding packages. The work is based on the energy consumption and does not consider any incentive mechanism. In [15], [16], the authors study an control problem where the source can control rate of the number of copies of the message by changing the probability of forwarding the message to a relay. The authors proved the optimal strategy for the source is of threshold type policy. The mean-field limit is presented when the number of nodes is very large. The mean-field not only helps the authors reduce the complexity of the problem but also lets them analyze the network with any size, i.e. with any number of nodes.

\section{Paper organization}

The paper is organized as follows: Section II presents our model and assumptions. We recall results which are necessary in this paper in Section III. The mean field model and mean field limits as well as the performance metrics are computed in Section IV for the case when the second threshold in infinite. An estimation to compute $a(t):=\theta(t) N$ for finite $\gamma$ is given in Section V.

\section{MODEL DESCRIPTION}

We consider a two-hop network with $N+2$ nodes with one source and one destination who are fixed and $N$ other nodes who play the role of relays. The source has many messages to send to its destination. $N$ relays move randomly in the network and may meet the source or the destination some time following an exponential distribution with rate $\lambda$, $\mu$ respectively. We assume that the mobility pattern of relays and the meeting times between the source and relay, between the destination and relay are i. i. d. When the source meets a relay, it will propose a message with a promised reward, $R$, for the first relay who successfully delivers the message to its destination. For a message, the fixed reward means the source proposes the same reward for all relays. A relay incurs a cost of receiving and transmitting a message of $C_{r}$ and $C_{d}$ respectively. Keeping a message costs $C_{s}$ per unit of time. For convenience, we denote $\bar{R}=R-C_{d}$. At anytime, a relay can accept or reject a message (when meeting the source), drop or keep when it has a message. There is no cost of dropping and rejecting a message. We assume that a relay only rejects or drops a message if the expected cost is positive. The two-hop network does not allow a relay to forward messages to other relays except to the destination. We also assume that a relay can store only one message at a time and it does not seek a new message while it is having one. That is, it only can accept a new message if it had rejected or transmitted or dropped the previous message. The source proposes messages to relays sequentially. The source and the relays have no feedback from the destination that the message has been transmitted or not. The source does not give any information about how many relays have the message. In our previous work [11], we proved that the optimal strategy of relays is of threshold-type: it accepts until the first threshold and it drop after the second threshold. We proved the uniqueness of the symmetric solution that is, all relays will play the same thresholds. The condition to have solution and the condition to have finite solution were also given. Based on that, we provided the formula to find the expected delay and the probability of success of the source for each message.

\section{Preliminaries}

In this section, we recall some results from [11] which will be used in the sequel. We recall one of the main theorems in [11] that gives the form of best-response policy. Theorem 3.1 in [11] stated that given the strategies of the other relays for message $k^{t h}$, the best-response policy $\pi_{i}^{*}(t ; x)$ at time $t$, state $x$ of relay $i$ is a threshold-type policy: there exists a $\theta_{k}^{i}$ and $\gamma_{k}^{i}>\theta_{k}^{i}$ such that $\pi_{i}^{*}\left(t ; m_{s}\right)=$ accept if and only if $t \leq \theta_{k}^{i}$, and $\pi_{k}^{*}(t ; 1)=d r o p$ if and only if $t>\gamma_{k}^{i}$. Moreover, $\left(\theta_{k}^{i}, \gamma_{k}^{i}\right)$ is the solution of:

$$
\begin{aligned}
& \gamma_{k}^{i}=\sup \left\{t: p_{k}^{i}(t)>\frac{C_{s}}{\mu\left(R-C_{d}\right)}\right\}, \\
& \theta_{k}^{i}=\sup \left\{t: C_{r}+G_{k}^{i}\left(t, \gamma_{k}^{i}\right)<0\right\},
\end{aligned}
$$

where by convention the supremum of the empty set is 0 and

$$
\begin{aligned}
G_{k}^{i}(a, b)= & \int_{a}^{b} \mu e^{-\mu(t-a)}\left(C_{s}(t-a)+\left(C_{d}-R\right) p_{k}^{i}(t)\right) d t \\
& +e^{-\mu(b-a)} C_{s}(b-a) .
\end{aligned}
$$

An immediate corollary stated that the type of policy at a Nash equilibrium is a threshold-type policy, that is, for a message $k$, there exist vectors $\theta_{k}$ and $\gamma_{k}$ such that relay $i$ uses a thresholdtype strategy with parameters $\left(\theta_{k}^{i}, \gamma_{k}^{i}\right)$.

In this paper, we consider the symmetric case. That is, we assume that all relays will play the same thresholds $\theta_{k}$ and $\gamma_{k}$ at equilibrium. In this case, the existence and the uniqueness of the symmetric solution was proved in [11]. To compute the thresholds for each message, we need to find the exact form of the probability that a relay fails to deliver message $k$ 
to the destination by time $t, p_{k}(t)$ used in (1) and (2). This probability can be computed as the follows:

$$
p_{k}(\tau)=V_{k}\left(\min \left(\theta_{k}, \tau\right), \min \left(\gamma_{k}, \tau\right)\right)^{N-1},
$$

where

$$
\begin{aligned}
V_{k}(x, y) & =1-\int_{\theta_{k-1}}^{x} \phi_{k}(s)\left(1-v_{x, y}(s)\right) d s \\
v_{x, y}(s) & =e^{-\lambda(x-s)}+\frac{\lambda}{\mu-\lambda} e^{-\mu y} e^{\lambda s}\left(e^{(\mu-\lambda) x}-e^{(\mu-\lambda) s}\right),
\end{aligned}
$$

with $\phi_{k}(s)$ be the density function of the event: "a relay is ready for message $k$ at time $s$ ". The expression above depends on the value of $\theta_{k-1}$. Therefore, by recursion, it requires all the knowledge of message 1 to message $k-1$ to do the analysis of message $k$ which gives us a challenge. The complexity also comes from the density function $\phi_{k}(s)$. We define $I_{k}(x, \tau)$ as the probability that a relay that comes into play at time $x$ will accept the $k^{\text {th }}$ message and will not be able to deliver it to the destination by time $\tau \in\left[\theta_{k}, \gamma_{k}\right]$. Therefore,

$$
I_{k}(x, \tau)=\frac{e^{-\mu \tau}}{\mu-\lambda} \lambda e^{\lambda x}\left(e^{(\mu-\lambda) \theta_{k}}-e^{(\mu-\lambda) x}\right)
$$

The density function $\phi_{k}(\tau)$ can be defined as follows. For $\tau \in\left[\theta_{k}, \gamma_{k}\right]$,

$$
\begin{aligned}
\phi_{k+1}(\tau)= & h_{1}\left(\theta_{k}\right) \delta_{\theta_{k}}(\tau)+\phi_{k}(\tau) \\
& +h_{2}\left(\theta_{k}\right) e^{\mu \theta_{k}}\left\{\mu e^{-\mu \tau}+e^{-\mu \gamma_{k}} \delta_{\gamma_{k}}(\tau)\right\}
\end{aligned}
$$

where

$$
\begin{aligned}
h_{1}\left(\theta_{k}\right) & =\int_{\theta_{k-1}}^{\theta_{k}} \phi_{k}(x)\left\{1-I_{k}\left(x, \theta_{k}\right)\right\} d x, \\
h_{2}\left(\theta_{k}\right) & =\int_{\theta_{k-1}}^{\theta_{k}} \phi_{k}(x) I_{k}\left(x, \theta_{k}\right) d x .
\end{aligned}
$$

The condition to have a symmetric Nash equilibrium with $\theta_{k}>0$ is

$$
\bar{R} \geq C_{r}+\frac{C_{s}}{\mu} .
$$

This Nash equilibrium is finite, i.e. $\theta_{k} \leq \gamma_{k}<\infty$ if and only if

$$
1+\mu \frac{C_{r}}{C_{s}}<\frac{(1+b)^{N}-1}{N b}
$$

where

$$
b=\frac{1}{\sigma \omega} \int_{\theta_{k-1}}^{\theta_{\min }} \phi_{k}(s)\left(e^{-\lambda\left(\theta_{\min }-s\right)}-e^{-\mu\left(\theta_{\min }-s\right)}\right) d s,
$$

with $\omega=\left(\frac{C_{s}}{\mu R}\right)^{1 /(N-1)}$ and $\sigma=\frac{\mu-\lambda}{\lambda}$ and $\theta_{\min }$ be the solution of

$$
1+\int_{\theta_{k-1}}^{\theta_{\min }} \phi_{k}(s)\left(e^{-\lambda\left(\theta_{\min }-s\right)}-1\right) d s=\omega,
$$

We denote $\hat{\theta}_{k}:=\theta_{k}-\theta_{k-1}$, the interval of time during which message $k$ is proposed by the source and $\hat{\gamma}_{k}:=\gamma_{k}-\theta_{k}$, the interval during which a relay does not drop message $k$.

\section{ASYMPTOTIC ANALYSIS}

In this section, we study the asymptotic performance of the network when the number of relays, $N$, is large. Let $\mathbf{Y}^{N}(\tau) \equiv$ $\left(Y_{1}^{N}(\tau), Y_{2}^{N}(\tau), \ldots, Y_{N}^{N}(\tau)\right)$ be a continuous-time stochastic process where $Y_{i}^{N}(\tau) \in\{0,1\}$ indicates whether relay $i$ has message at time $\tau$ or not.

Consider the discrete-time embedding of $\mathbf{Y}$ and $\theta_{k}$ defined by $\mathbf{X}^{N}(k)=\mathbf{Y}\left(\theta_{k}\right)$. The process $\mathbf{X}^{N}$ lives on $\mathcal{S}^{N}=\{0,1\}^{N}$. Here $X_{n}^{N}(k)$ is the state of relay $n$ at $\theta_{k-1}$. If $X_{n}^{N}(k)=1$, then relay $n$ has a message at the release time of message $k$, otherwise it is free to accept message $k$ from the source. With this definition, the duration of time-slot $k$ is of length $\hat{\theta}_{k}$.

The process $\mathbf{X}^{N}$ is a discrete-time Markov chain only if $\hat{\gamma}_{k}$ is infinite for all $k$. Otherwise, we also need to keep track of the identity of the message held by a relay as well as the $\hat{\gamma}$ of that message to be able to define the dynamics of $\mathbf{X}^{N}$.

We now give the condition to have infinite $\hat{\gamma}_{k}$ when $N$ is large. Interestingly, the condition does not depend on $k$. For this, we first need the following results which shall be invoked later as well.

Lemma 4.1:

$$
\lim _{N \rightarrow \infty} N \hat{\theta}_{k}=c_{k}
$$

\section{Proof: See Appendix A.}

The above result states that the duration for which the source proposes message $k$, that is $\hat{\theta}_{k}$ is $O(1 / N)$ as $N \rightarrow \infty$. The intuition behind this result is the following. When the number of relays is large, in order to observe a change in the occupancy measure of any state, we need to look at messages that have sequence numbers of $O(N)$. The intuitive reasoning is that a message is profitable to accept only if there are a finite number of relays that are competing to deliver this message. Otherwise, the probability of success of a relay will be zero, and it will not accept the message. Since, on an average, there will be at least $\lambda \hat{\theta}_{k} N M_{0}^{N}(k)$ relays that will pick message $k, \hat{\theta}_{k}$ should be $0(1 / N)$ in order for the average number of competing relays to be finite for each message. Thus, we need to look at messages $k=t N$ to observe changes in the occupancy measure.

Proposition 4.1: When $N$ is large, there exists a symmetric Nash equilibrium with $\hat{\theta}_{k}>0$ if and only if $\bar{R} \geq C_{r}+\frac{C_{s}}{\mu}$. This solution is finite if and only if

$$
1+\mu \frac{C_{r}}{C_{s}}<\frac{\frac{\mu \bar{R}}{C_{s}}-1}{\ln \left(\frac{\mu \bar{R}}{C_{s}}\right)} .
$$

\section{Proof: See Appendix B.}

The RHS of the condition above increases in $\bar{R}=R-C_{d}$. That means for a small enough reward $R$, we will get a infinite $\hat{\gamma}_{k}$.

In the rest of this section, we shall assume that the condition (15) is satisfied so that $\mathbf{X}^{N}$ is a discrete-time Markov chain. 
We observe that the transitions of each $X_{n}^{N}$ are independent from that of the others. The transition probabilities between the states are

$$
\begin{aligned}
p_{0,1}= & \left(1-e^{-\lambda \hat{\theta}_{k}}\right) \cdot e^{-\mu \hat{\theta}_{k}} \\
= & \lambda \hat{\theta}_{k}+o\left(\hat{\theta}_{k}\right), \\
p_{1,0}= & \left(1-e^{-\mu \hat{\theta}_{k}}\right) \cdot\left(e^{-\lambda \hat{\theta}_{k}}\right. \\
& \left.+\left(1-e^{-\lambda \hat{\theta}_{k}}\right) \cdot\left(1-e^{-\mu \hat{\theta}_{k}}\right)\right) \\
= & \mu \hat{\theta}_{k}+o\left(\hat{\theta}_{k}\right)
\end{aligned}
$$

The expression for $p_{1,0}$ has the following explanation. The transition from state 1 to state 0 happens if the relay meets the destination for the first time to deliver the message that it has. Then, to remain in state 0 it should either not meet the source until the end of the interval $\hat{\theta}_{k}$ or if it meets the source before the end of this interval then it should again meet the destination.

\section{A. Mean-field limit}

In [17], a general framework for showing the convergence of discrete-time Markov chains to mean-field dynamics is given. Following their steps, let $M^{N}(k)$ be the occupancy measure which is the vector of frequencies of state $s \in \mathcal{S}$ at time $t$,

$$
M_{s}^{N}(k)=\frac{1}{N} \sum_{n=1}^{N} \mathbb{1}_{\left\{X_{n}^{N}(k)=s\right\}} .
$$

We have $M^{N}(k) \in \Delta:=\left\{\mathbf{m} \in \mathbb{R}^{2}, m_{1}+m_{2}=1, m_{1}, m_{2} \geq\right.$ $0\}$.

Define a time rescaled process $\bar{M}^{N}(t)$ as

$$
\left\{\begin{array}{l}
\bar{M}_{s}^{N}(t)=M_{s}^{N}(t / N) \text { for all } t \\
\left.\bar{M}_{s}^{N}(\tau) \text { is affine on } \tau \in[t, t+1 / N)\right]
\end{array}\right.
$$

Let $\hat{a}(t)=N \hat{\theta}_{t N}$ be the rescaled interval during which message $k=t N$ is proposed . Let $\hat{a}_{0}$ be the rescaled interval for the first message when all the relays are available to compete for this message (see [12] for its computation). The following proposition gives the mean-field limit of the rescaled occupancy measure.

Proposition 4.2: Let $m_{0}(t)$ be the solution of the differential equation

$$
\dot{m}_{0}(t)=-\lambda m_{0}(t) \hat{a}(t)+\mu\left(1-m_{0}(t)\right) \hat{a}(t),
$$

with $\hat{a}(t)=\frac{\hat{a}_{0}}{m_{0}(t)}$ and $m_{0}(0)=m$. Assume $M_{s}^{N}(0) \rightarrow m$ in probability as $N \rightarrow \infty$. Then, for all $t>0$, as $N \rightarrow \infty$,

$$
\sup _{0 \leq \tau \leq t}\left\|\bar{M}_{0}^{N}(\tau)-m_{0}(\tau)\right\| \rightarrow 0
$$

in probability.

Proof: The proof is based upon the verification of conditions in [17] and is given in Appendix C.

The above proposition gives the fraction of relays that are available to compete for message $t$. The following result tells the duration for which this message will be proposed by the source.

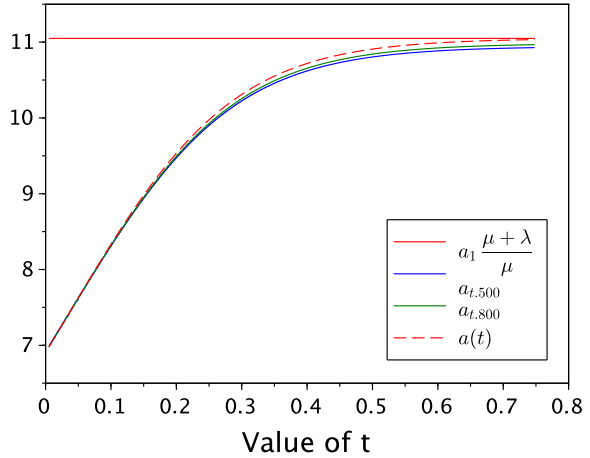

Fig. 1. The behavior of $\hat{a}(t)$ when $\mu=0.5, \lambda=0.3$ and $N=500 ; 800$.

Proposition 4.3: Let $\hat{a}(0)=\hat{a}_{0} / m_{0}(0)$. Then,

$$
\hat{a}(t)=\frac{\hat{a}(0)}{\alpha+(1-\alpha) u},
$$

where $u$ is the solution of the following equation

$$
-\beta \hat{a}(0) t=\alpha \ln (u)+(1-\alpha) u+\alpha-1,
$$

where $\alpha=\frac{\mu}{\mu+\lambda}, \beta=\mu+\lambda$.

Proof: Equation (20) can be rewritten in terms of $\hat{a}$ to get the following differential equation

$$
\frac{\dot{\hat{a}}(t)}{\hat{a}^{2}(t)}=(\lambda+\mu) \hat{a}(0)+\mu \hat{a}(t) .
$$

The solution of this differential equation is given by

$$
\hat{a}(t)=\frac{\hat{a}(0)}{\alpha+(1-\alpha) e^{-\beta \int_{0}^{t} \hat{a}(s) d s}}
$$

Let $u=e^{-\beta \int_{0}^{t} \hat{a}(s) d s}$, then $\dot{u}=-\beta \hat{a}(t) u$. Plugging this substitution into the above equation and taking the integral we will gives the claimed result.

Using this result, we can also compute the time at which message $t$ will be released by the source.

Corollary 4.1: Let $a(t)=\lim _{N \rightarrow \infty} \theta_{t N}$ be the release time of message $t$. Then,

$$
a(t)=-\frac{1}{\beta} \ln (u),
$$

where $u$ is the solution of

$$
-\beta \hat{a}(0) t=\alpha \ln (u)+(1-\alpha) u+\alpha-1 .
$$

Proof: The claim follows by noting that message $t$ is released at time $\int_{0}^{t} \hat{a}(s) d s$ and using the definitions in the above proposition.

We check numerically that the mean-field ODE is gives a good approximation for $\hat{a}(t)$ for finite $N$. First, we let $\mu<\lambda$, and take $\mu=0.5, \lambda=0.3$ (Figure 1) and in Figure 2 the comparison is done for $\mu=\lambda=0.4$. 


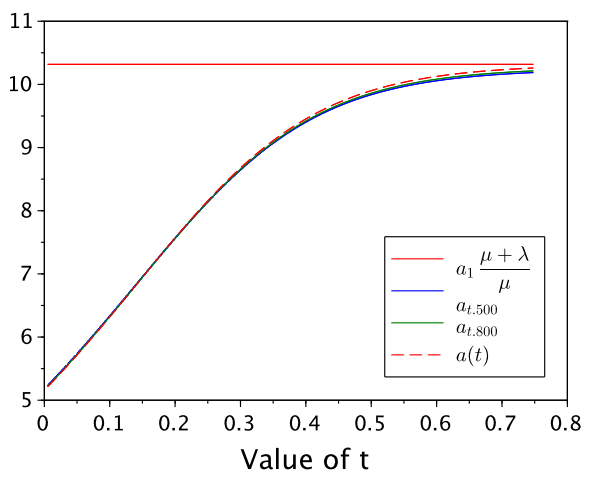

Fig. 2. The behavior of $\hat{a}(t)$ when $\mu=\lambda=0.4$ and $N=500 ; 800$.

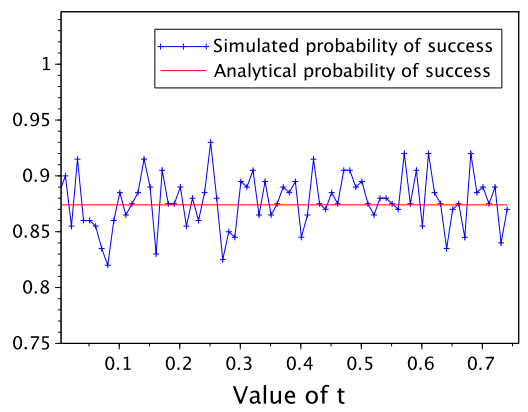

Fig. 3. The simulated probability and its analytical value with $N=800$, $\mu=0.5, \lambda=0.3$.

\section{B. Performance metrics}

In this part, we will find the probability of success of message number $k=t N$ for $N$ is large as well as its expected delay. The following proposition present the probability of success and the delay when $N$ is large.

Proposition 4.4: If $k=t N$ and $N$ is large, we have the probability of success of message $k^{t h}$ (denoted by $\xi(t)$ ) will be

$$
\xi(t)=1-e^{-\lambda \hat{a}_{0}}, \quad \forall t .
$$

We let $D(t)$ be the delay provided that at least one copy of message has reached the destination. Then the expected value of $D(t)$ is

$$
\mathbb{E}(D(t) \mid D(t)<\infty)=\frac{e^{-\lambda \hat{a}_{1}}}{\mu\left(1-e^{-\lambda \hat{a}_{1}}\right)} \int_{0}^{1} \frac{e^{\lambda \hat{a}_{1} u}-1}{u} d u .
$$

Proof: See Appendix D.

The probability of success and the expected delay do not depend on $t$ since the average number of relays who have the message during the time between two consecutive $\theta$ are the same when $N$ is large. Figures 3 and 4 shows that the simulated probability of success and the simulated delay are close to the analytical results.

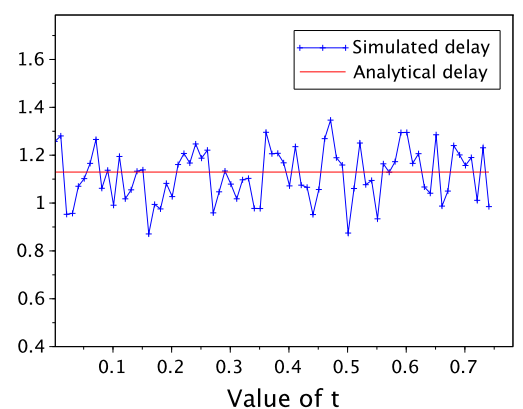

Fig. 4. The simulated delay and its analytical value with $N=800, \mu=$ $0.5, \lambda=0.3$.

\section{Estimation IN THE FINITE $\gamma$ CASE}

When $\gamma_{k}$ is finite, it is more complicated since the mean field model is no longer a Markov chain. Therefore, in order to find the limit, we need to know exactly how many relays have message $k$ for all $k$ and how long they will keep that message counting from current time. That is not an easy job. In this section, we just give an estimation of $m_{0}(t)$.

In the following Proposition, we present an observation of $\hat{a}(t)$ and $\hat{\gamma}(t)$.

Proposition 5.1: When $\gamma_{k}$ is finite, we have for all $t$,

$$
\begin{aligned}
\hat{a}(t) & =\frac{\hat{a}_{1}}{h_{1}(t)}, \\
\hat{\gamma}(t) & =\hat{\gamma}_{1} .
\end{aligned}
$$

Proof: When $\gamma_{k}$ is finite, some following estimations still hold.

$$
\begin{aligned}
h_{2}(t) & \approx \lambda \hat{\theta}(t) h_{1}(t), \\
\int_{0}^{\hat{\theta}(t)} \phi_{t}(s) d s & \approx h_{1}(t), \\
p_{t}(\hat{\gamma}(t))^{\frac{N}{N-1}} & \approx e^{-\lambda \hat{a}(t) h_{1}(t)\left(1-e^{-\mu \hat{\gamma}(t)}\right)}, \\
p_{t}(\hat{\theta}(t))^{\frac{N}{N-1}} & \approx 1 .
\end{aligned}
$$

Therefore, we have the equations system to find $\hat{\gamma}(t)$ and $\hat{\theta}(t)$ as follows

$$
\begin{gathered}
e^{-\lambda \hat{a}(t) h_{1}(t)\left(1-e^{-\mu \hat{\gamma}(t)}\right)} \approx \frac{C_{s}}{\mu \bar{R}}, \\
C_{r}-\frac{C_{s}}{\mu} \frac{\log (v)}{\lambda \hat{a}(t) h_{1}(t)}+\frac{\bar{R}}{\lambda \hat{a}(t) h_{1}(t)}=0,
\end{gathered}
$$

where $v=\frac{C_{s}}{\mu \bar{R}}$. Eq. (35) means when $t$ is closed to 0 , we will get $\hat{a}_{1}$, hence for all $t$ we have

$$
\hat{a}(t)=\frac{\hat{a}_{1}}{h_{1}(t)} .
$$

Plugging this result into the Eq. (34), we also get

$$
\hat{\gamma}(t)=\hat{\gamma}_{1}, \quad \text { for all } t
$$




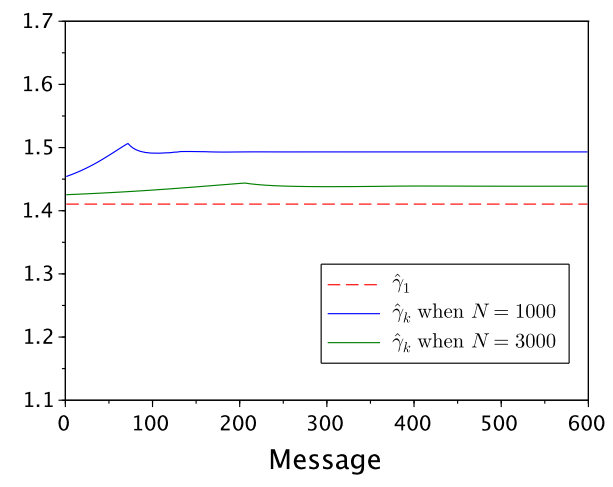

Fig. 5. The value of $\hat{\gamma}_{1}$ and $\hat{\gamma}_{k}$ with $N=1000 ; 3000, \mu=0.8, \lambda=0.4$. We see that when $N$ is large, the duration of time that a relay keeps a message is the same for all messages.

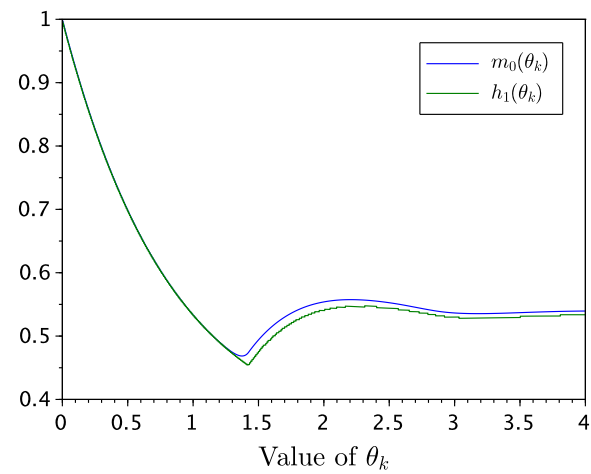

Fig. 6. The function $m_{0}$ and function $h_{1}$ with $N=3000, \mu=0.8, \lambda=0.4$.

We also have $h_{1}(t) \approx m_{0}(a(t))$ where $a(t)=\int_{0}^{t} \hat{a}(s) d s$. Hence, we only need to estimate the function $m_{0}(x)$.

For convenience, we chance the time-scale and give the ODE for $m(a(t)) \equiv m_{0}(x)$. The change in $m_{0}(x)$ in a $\delta x$ will be the difference between the ones who meet the destination or drop at time $x$ and the ones who are available to accept a new message. At any time $x$, there are $\lambda m_{0}\left(x-\hat{\gamma}_{1}\right) e^{-\mu \hat{\gamma}_{1}}$ relays will drop their messages because the reach the second threshold. In addition there will be $m_{0}(x) \lambda$ who will change state to 1 and $\left(1-m_{0}(x) \mu\right)$ who will change state to 0 . Then, we have

$\frac{d m_{0}(x)}{d x}=-\lambda m_{0}(x)+\mu\left(1-m_{0}(x)\right)+\lambda m_{0}\left(x-\hat{\gamma}_{1}\right) e^{-\mu \hat{\gamma}_{1}}$.

Solving (38) gives us $m_{0}(x)$, then plugging into (28), we will get $\hat{a}(t)$ for all $t$.

Figure 5 confirms that when $N$ is large, the duration of time that a relay keeps a message is the same for all messages. Figure 7 verifies that the estimated $\hat{a}(t)$ is close to the analytical value of $a(t)$.

For the probability of success and the delay, we do similarly

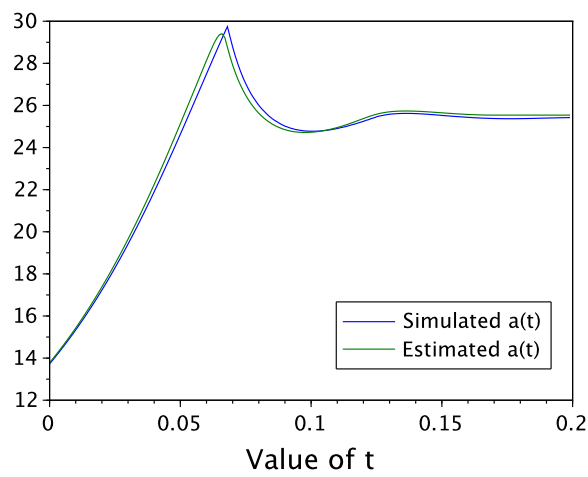

Fig. 7. The estimated $\hat{a}(t)$ and the analytical $a(t)$ with $N=3000, \mu=$ $0.4, \lambda=0.8$.

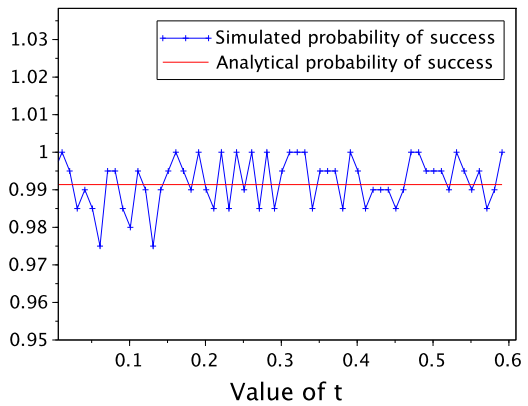

Fig. 8. The simulated probability of success and its analytical value with $N=1000, \mu=0.8, \lambda=0.4$.

to the infinite $\gamma(t)$. We have the average number of relays who have a message stays the same and equals to that number of the first message. So that we will get the same probability of success and the delay for all message.

Proposition 5.2: When $\gamma_{k}$ is finite, for all $t$, the probability of success and the delay are

$$
\begin{aligned}
\xi(t) & =1-\frac{C_{s}}{\mu \bar{R}}, \\
\mathbb{E}(D(t) \mid D(t)<\gamma(t)) & =\frac{e^{-\lambda \hat{a}_{1}}}{\mu(1-v)} \int_{e^{-\mu \hat{\gamma}_{1}}}^{1} \frac{e^{\lambda \hat{a}_{1} u}-1}{u} d u
\end{aligned}
$$

where $v=\frac{C_{s}}{\mu \bar{R}}$.

As in the case $\gamma(t)=\infty$, we have the same delay and probability of success for all messages as in Figure 8.

\section{CONCLUSION}

We consider a network with $N$ relays and one pair of source-destination. The source is backlogged and proposes a fixed reward for each message. We study the mean-field limit of this game when the second threshold is infinite and show that in this limit each message is proposed for a duration of $O(1 / N)$. We show that the fraction of relays without a message converges in the mean field limit to the solution of 
an ODE. Based on that limit, we find the formula to compute various performance metrics such as probability of success and the mean delay. It is shown that the probability of success is the same for all messages.

When the second threshold is finite, the dynamics are no longer Markovian and we propose an ODE approximation which numerically gives a good match.

\section{REFERENCES}

[1] M. Ito, H. Nishiyama, and N. Kato, "A novel routing method for improving message delivery delay in hybrid dtn-manet networks," in 2013 IEEE Global Communications Conference (GLOBECOM), Dec 2013, pp. 72-77.

[2] A. Sudarsono and T. Nakanishi, "An implementation of secure data exchange system with multi-hop routing in wireless delay tolerant network using attribute-based encryption," in 2015 Third International Symposium on Computing and Networking (CANDAR), Dec 2015, pp. 470-476.

[3] M. Malathi and S. Jayashri, "Design and performance of dynamic trust management for secure routing protocol," in 2016 IEEE International Conference on Advances in Computer Applications (ICACA), Oct 2016, pp. 121-124.

[4] J. Wu, J. Wang, L. Liu, M. Tanha, and J. Pan, "A data forwarding scheme with reachable probability centrality in dtns," in 2016 IEEE Wireless Communications and Networking Conference, April 2016, pp. 1-6.

[5] N. Basilico, M. Cesana, and N. Gatti, "Algorithms to find two-hop routing policies in multiclass delay tolerant networks," IEEE Transactions on Wireless Communications, vol. 15, no. 6, pp. 4017-4031, June 2016.

[6] Y. Cai, Y. Fan, and D. Wen, "An incentive-compatible routing protocol for two-hop delay-tolerant networks," IEEE Transactions on Vehicular Technology, vol. 65, no. 1, pp. 266-277, Jan 2016.

[7] N. Torabkhani and F. Fekri, "Delay analysis of bursty traffic in finitebuffer disruption-tolerant networks with two-hop routing," in 2013 IEEE International Conference on Sensing, Communications and Networking (SECON), June 2013, pp. 541-549.

[8] A. Al-Hanbali, P. Nain, and E. Altman, "Performance of ad hoc networks with two-hop relay routing and limited packet lifetime," Performance Evaluation, vol. 65, no. 6-7, pp. 463-483, June 2008.

[9] T. Seregina, O. Brun, R. Elazouzi, and B. Prabhu, "On the design of a reward-based incentive mechanism for delay tolerant networks," IEEE Transactions on Mobile Computing, vol. 16, no. 2, pp. 453-465, 2017.

[10] E. Altman, Competition and Cooperation between Nodes in Delay Tolerant Networks with Two Hop Routing, 2009.

[11] T. T. H. Nguyen, O. Brun, and B. Prabhu, "Performance of a fixed reward incentive scheme for two-hop DTNs with competing relays (long version)," Aug. 2017, this is the long version of the NetEcon paper http://netecon.eurecom.fr/NetEcon2016/papers/Nguyen.pdf which is also in HAL https://hal.archives-ouvertes.fr/hal-01365939. [Online]. Available: https://hal.laas.fr/hal-01575320

[12] T. T. H. Nguyen, O. Brun, and B. J. Prabhu, "Performance of a fixed reward incentive scheme for two-hop dtns with competing relays: Short talk," SIGMETRICS Perform. Eval. Rev., vol. 44, no. 3, pp. 39-39, Jan. 2017. [Online]. Available: http://doi.acm.org/10.1145/3040230.3040244

[13] L. Sassatelli, A. Ali, M. Panda, T. Chahed, and E. Altman, "Reliable Transport in Delay-Tolerant Networks With Opportunistic Routing," IEEE Transactions on Wireless Communications, vol. 13, no. 10, pp. 5546-5557, Oct. 2014. [Online]. Available: https://hal.inria.fr/hal01101545

[14] S. Eshghi, M. H. R. Khouzani, S. Sarkar, N. B. Shroff, and S. S Venkatesh, "Optimal energy-aware epidemic routing in dtns," IEEE Transactions on Automatic Control, vol. 60, no. 6, pp. 1554-1569, June 2015.

[15] F. D. Pellegrini, E. Altman, and T. Basar, "Optimal monotone forwarding policies in delay tolerant mobile ad hoc networks with multiple classes of nodes," in 8th International Symposium on Modeling and Optimization in Mobile, Ad Hoc, and Wireless Networks, May 2010, pp. 497-504.

[16] E. Altman, T. Baar, and F. D. Pellegrini, "Optimal monotone forwarding policies in delay tolerant mobile ad-hoc networks," Performance Evaluation, vol. 67, no. 4, pp. 299 - 317, 2010, performance Evaluation Methodologies and Tools: Selected Papers from VALUETOOLS 2008. [Online]. Available: http://www.sciencedirect.com/science/article/pii/S0166531609001187
[17] M. Benaïm and J.-Y. Le Boudec, "A class of mean field interaction models for computer and communication systems," Perform. Eval., vol. 65, no. 11-12, pp. 823-838, Nov. 2008. [Online]. Available: http://dx.doi.org/10.1016/j.peva.2008.03.005

\section{APPENDIX A}

\section{PROOF OF LEMMA 4.1}

From (3), we observe that $p_{k}(\tau)$ should be strictly positive in the limit $N \rightarrow \infty$ for a relay to accept message $k$. From (4) and (5), this is equivalent to

$$
\int_{\theta_{k-1}}^{\theta_{k}} \phi_{k}(s)\left(1-v_{\theta_{k}, \tau}(s)\right) d s=O(1 / N) .
$$

which is equivalent to

$$
\int_{0}^{\hat{\theta}_{k}} \phi_{k}\left(s+\theta_{k-1}\right)\left(1-v_{\theta_{k}, \tau}\left(s+\theta_{k-1}\right)\right) d s=O(1 / N) .
$$

From the definition of $v$ (see (5)), for $\hat{\theta}_{k} \rightarrow 0$,

$$
v_{\theta_{k}, \tau}\left(s+\theta_{k-1}\right)=1-\lambda\left(\hat{\theta}_{k}-s\right)\left(1-e^{-\mu\left(\tau-\theta_{k}\right)}\right)+o\left(\hat{\theta}_{k}\right) .
$$

Using the definition of $\phi_{k}$ is (7), we get the following asymptotics for the LHS of (40)

$$
\begin{array}{r}
\int_{0}^{\hat{\theta}_{k}} \phi_{k}\left(s+\theta_{k-1}\right)\left(1-v_{\theta_{k}, \tau}\left(s+\theta_{k-1}\right)\right) d s=h_{1}\left(\theta_{k-1}\right) \lambda \hat{\theta}_{k} \\
\cdot\left(1-e^{-\mu\left(\tau-\theta_{k}\right)}\right)+o\left(\hat{\theta}_{k}\right) .
\end{array}
$$

Next, we shall argue that $h_{1}\left(\theta_{k}\right)$ is $O(1)$ which will then imply that $\hat{\theta}_{k}$ has to be $O(1 / N)$ for (40) to hold. Consider the meanfield limit of the continuous time process $\mathbf{Y}$. When $\hat{\gamma}_{k}=\infty$, it can be seen that the scaled process $y_{1}(s)=\frac{1}{N} \sum_{i=1}^{N} Y_{i}(s)$ converges to the ODE

$$
\dot{y}_{1}(s)=\lambda y_{0}(s)-\mu y_{1}(s) .
$$

Here $y_{1}(t)$ is the fraction of relays that have a message at time $s$. This ODE has a unique solution for which $y_{1}(s) \in(0,1), \forall s$ if $y_{1}(0) \in(0,1)$. Thus, for all time $s$ the fraction of nodes available to compete for a message is strictly positive. The probability that a relay becomes available to take message $k$ is $\int_{\theta_{k-1}}^{\theta_{k}} \phi_{k}(s) d s$ which, from (7), tends to $h_{1}\left(\theta_{k-1}\right)$ when $\hat{\theta}_{k} \rightarrow 0$. Thus, $h_{1}$ is $O(1)$ and $\hat{\theta}_{k}$ is $O(1 / N)$.

\section{APPENDIX B}

\section{ProOF OF Proposition 4.1}

Let us consider message $t N$, and let $\theta_{\min }(t)$ be $\theta_{\min }$ of message $k=t N$. We assume that when $N$ is large, the $\theta(t)-$ $\theta_{\text {min }} \approx \frac{a_{\min }(t)}{N}$. Therefore the Eq. (13) can be approximated as $1-h_{1}(t) \lambda \hat{\theta}_{\min }(t)=\omega$, hence

$$
\left(1-\frac{h_{1}(t) \lambda a_{\min }(t)}{N}\right)^{N-1}=\frac{C_{s}}{\mu \bar{R}} .
$$

When $N$ is large, the LHS of that equation tends to $e^{-\lambda a_{\min }(t) h_{1}(t)}$. Therefore, when $N$ is large, $a_{\min }(t)$ will be

$$
a_{\text {min }}(t)=\frac{1}{h_{1}(t) \lambda} \ln \left(\frac{\mu \bar{R}}{C_{s}}\right) \text {. }
$$


Similarly, we have the approximation for $b$. First, we observe that $\lim _{N \rightarrow \infty} \omega=1$. From Eq. (12), $b$ can be approximated as

$$
b \approx \frac{\lambda}{\mu-\lambda}(\mu-\lambda) h_{1}(t) \theta_{\min }(t)=h_{1}(t) \lambda \theta_{\min }(t) .
$$

Hence, we can get the limitation of $N b$ and $(1+b)^{N}$ when $N$ is large,

$$
\lim _{N \rightarrow \infty} \frac{(1+b)^{N}-1}{N b}=\frac{e^{h_{1}(t) \lambda a_{\min }(t)}-1}{h_{1}(t) \lambda a_{\min }(t)} .
$$

Plug the value of $a_{\min }(t)$ into the Eq. (11) we get

$$
1+\mu \frac{C_{r}}{C_{s}}<\frac{\frac{\mu \bar{R}}{C_{s}}-1}{\ln \left(\frac{\mu \bar{R}}{C_{s}}\right)} .
$$

\section{APPENDIX C}

\section{PROOF OF PROPOSITION 4.2}

First, we shall show that $\hat{a}(t)=a_{0} / m(t)$. One computes $\theta_{k}$ from (2) and (3). When $N$ is large, and assuming $k=t N$ and $\hat{\gamma}_{k}=\infty$, one can show that

$$
\begin{aligned}
& G_{k}\left(\theta_{k}, \gamma_{k}\right)=C_{r}+\frac{C_{s}}{\mu}\left(1-e^{-\hat{\gamma}_{k}}\right) \\
& -\frac{(C d-R)}{h_{2}\left(\theta_{k}\right)} \frac{1}{N}\left(p_{k}(\infty)^{N /(N-1)}-p_{k}\left(\theta_{k}\right)^{N /(N-1)}\right) .
\end{aligned}
$$

With some abuse of notation, we shall use $h_{i}(t)$ to denote $h_{i}\left(\theta_{t N}\right)$. From (7), one has the approximation $h_{2}(t) \approx$ $h_{1}(t) I_{t}\left(\theta_{t N}, \theta_{t N}\right) \approx h_{1}(t) \lambda \hat{a}(t) / N$. It can be shown that

$$
p_{k}(\infty)^{N /(N-1)} \approx\left(1-\frac{\left.\lambda \hat{a}(t) h_{1}(t)\right)}{N}\right)^{N} \underset{N \rightarrow \infty}{\longrightarrow} e^{-\lambda \hat{a}(t) h_{1}(t)} .
$$

and $p_{k}\left(\theta_{k}\right)^{N /(N-1)} \rightarrow 1$. Thus, any solution of (44) has the form $a \hat{(t)} h_{1}(t)=c$, a constant. As argued in the proof of Lemma $4.1 h_{1}(t)=m_{0}(t)$. Denoting $\hat{a}_{0}$ to the constant when $m_{0}(t)=1$ we get the desired relationship.

Next, we check that the conditions in [17] are verified. Let $f^{N}\left(m_{0}\right)$ be the drift function which is the expected change to $M_{0}^{N}$ in one time slot,

- Intensity vanishes at a rate $\epsilon(N)$ : We take $\epsilon(N)=\frac{1}{N}$. We need to prove that

$$
\lim _{N \rightarrow \infty} \frac{f^{N}\left(m_{0}\right)}{\epsilon(N)}=f\left(m_{0}\right), \text { exists for all } m_{0} \in(0,1) .
$$

From (16) and (17), and Lemma 4.1, we have

$$
\frac{f^{N}\left(m_{0}\right)}{\epsilon(N)}=N m_{1} \frac{\mu \hat{a}(t)}{N}-m_{0} \frac{\lambda \hat{a}(t)}{N} .
$$

Hence,

$$
\lim _{N \rightarrow \infty} \frac{f^{N}\left(m_{0}\right)}{\epsilon(N)}=m_{1} \mu a(t)-m_{0} \lambda a(t) .
$$

- Second moment of number of object transition per time slot: There are two types of transitions from 0 to 1 and from 1 to 0 . The total number of transitions of the first type has a Binomial distribution with parameters $N_{i}$ and $\lambda \hat{a}(t) / N_{i}$ where $N_{i}$ is the number of relays with no messages. This tends to Poisson distribution that has a finite second moment. The same argument holds for the second type of transitions.

- $f^{N}(m)$ is a smooth function of $\frac{1}{N}$ and $m_{0}$ : The function $m_{1} \frac{\mu \hat{a}(t)}{N}-m_{0} \frac{\lambda \hat{a}(t)}{N}$ with $\hat{a}(t)=1 / m_{0}$ is smooth in $1 / N$ and in $m_{0}$.

\section{APPENDIX D}

ProOF OF PROPOSITION 4.4 is

From [11], we have the probability of success when $\gamma=\infty$

$$
\xi_{k}=1-\left(1-\int_{\theta_{k-1}}^{\theta_{k}} \phi_{k}(s)\left(1-e^{-\lambda\left(\theta_{k}-s\right)}\right) d s\right)^{N} .
$$

We remark that for $k=t N$, we have $m_{0}(\theta(t))=\frac{\hat{a}_{1}}{a(t)}$. Moreover, we also have

$$
\begin{aligned}
\xi_{k} & =1-\left(1-\int_{\theta_{k-1}}^{\theta_{k}} \phi_{k}(s)\left(1-e^{-\lambda\left(\theta_{k}-s\right)}\right) d s\right)^{N} \\
& =1-\left(1-m_{0}(\theta(t))\left(1-e^{-\lambda \hat{\theta}(t)}\right)\right)^{N}, \\
& =1-\left(1-\lambda \frac{\hat{a}_{1}}{N}\right)^{N} .
\end{aligned}
$$

Therefore,

$$
\xi(t)=\lim _{N \rightarrow \infty} 1-\left(1-\lambda \frac{\hat{a}_{1}}{N}\right)^{N}=1-e^{-\lambda \hat{a}_{1}} .
$$

For the delay, we have, for all $k$ the expected delay given that at least one copy of message has reached the destination is

$$
\frac{1}{\zeta_{k}} \int_{\theta_{k-1}}^{\gamma_{k}}\left(V_{k}\left(\min \left(s, \theta_{k}\right), s\right)^{N}-V_{k}\left(\theta_{k}, \gamma_{k}\right)^{N}\right) d s
$$

We now estimate the $V_{k}\left(\min \left(s, \theta_{k}\right), s\right)^{N}$ where $k=t N$ and $N$ is large. We have, for all $s \geq \theta(t)$,

$$
\begin{aligned}
V_{k}(s, s)= & 1-\int_{\theta(t)}^{\infty} \phi_{t}(y)\left(1-e^{-\lambda(\theta(t)-y)}\right. \\
& \left.-\frac{\lambda}{\mu+\lambda} e^{-\mu s} e^{\lambda y}\left(e^{(\mu-\lambda) \theta(t)}-e^{(\mu-\lambda) y}\right)\right) d y \\
= & 1-m_{0}(\theta(t)) \lambda \hat{\theta}(t)\left(1-e^{-\mu(s-\theta(t))}\right) \\
= & 1-\frac{\hat{a}_{1}}{N} \lambda\left(1-e^{-\mu(s-\theta(t))}\right) .
\end{aligned}
$$

Therefore, we have

$\lim _{N \rightarrow \infty}\left(1-\frac{\hat{a}_{1}}{N} \lambda\left(1-e^{-\mu(s-\theta(t))}\right)\right)^{N}=e^{-\lambda \hat{a}_{1}\left(1-e^{-\mu(s-\theta(t))}\right) .}$

Hence,

$\mathbb{E}(D(t) \mid D(t)<\infty)=\int_{\theta(t)}^{\infty} \frac{e^{-\lambda \hat{a}_{1}}}{\xi(t)}\left(e^{\lambda \hat{a}_{1} e^{-\mu(s-\theta(t))}}-1\right) d s$,

and by changing variable we get the stated result. 